\title{
Molecularly-Defined Hippocampal Inputs Regulate Population Dynamics in the Prelimbic Cortex to Suppress Context Fear Memory Retrieval
}

\author{
Henry L. Hallock ${ }^{1}$, IV Henry M. Quillian ${ }^{1}$, Kristen R. Maynard ${ }^{1}$, Yishan Mai ${ }^{1}$, Huei-Ying \\ Chen ${ }^{1}$, Gregory R. Hamersky ${ }^{1}$, Joo Heon Shin ${ }^{1}$, Brady J. Maher ${ }^{1,2,3}$, Andrew E. \\ Jaffe $^{1,2,4,5,6,7}$, Keri Martinowich $1,2,3,{ }^{*}$ \\ ${ }^{1}$ The Lieber Institute for Brain Development, Baltimore, MD \\ 2Department of Psychiatry, The Johns Hopkins University School of Medicine, Baltimore, MD \\ ${ }^{3}$ Department of Neuroscience, The Johns Hopkins University School of Medicine, Baltimore, MD \\ ${ }^{4}$ Department of Mental Health, Johns Hopkins Bloomberg School of Public Health, Baltimore, MD \\ ${ }^{5}$ Department of Biostatistics, Johns Hopkins Bloomberg School of Public Health, Baltimore, MD \\ ${ }^{6}$ Center for Computational Biology, Johns Hopkins University, Baltimore, MD \\ ${ }^{7}$ McKusick-Nathans Institute for Genetic Medicine, Johns Hopkins School of Medicine, Baltimore, \\ $\mathrm{MD}$
}

\begin{abstract}
Background: Context-fear memory dysregulation is a hallmark symptom of several neuropsychiatric disorders, including generalized anxiety disorder (GAD) and post-traumatic stress disorder (PTSD). The hippocampus and prelimbic subregion (PrL) of the medial prefrontal cortex (mPFC) have been linked with context fear memory retrieval in rodents, but the mechanisms by which hippocampal-prelimbic circuitry regulates this process remains poorly understood.
\end{abstract}

Methods: Spatial and genetic targeting of hippocampal-prelimbic circuitry for RNA-sequencing $(n=31)$, chemo-genetic stimulation $(n=44)$, in vivo calcium imaging $(n=20)$, ex vivo electrophysiology $(n=8)$, and molecular regulation of plasticity cascades during fear behavior (context fear retrieval; $n=16$ ).

Results: We show that hippocampal neurons with projections to the PrL (vHC-PrL projectors) are a transcriptomically-distinct sub-population compared to adjacent non-projecting neurons, and show complementary enrichment for diverse neuronal processes and CNS-related clinical gene

*Correspondence: Keri Martinowich, 855 N. Wolfe St., \#382, Baltimore, MD 21205 keri.martinowich@libd.org.

Financial Disclosure Statement

The authors report no biomedical financial interests or potential conflicts of interest.

Publisher's Disclaimer: This is a PDF file of an unedited manuscript that has been accepted for publication. As a service to our customers we are providing this early version of the manuscript. The manuscript will undergo copyediting, typesetting, and review of the resulting proof before it is published in its final form. Please note that during the production process errors may be discovered which could affect the content, and all legal disclaimers that apply to the journal pertain. 
sets. We further show that stimulation of this population of vHC-PrL projectors suppresses context fear memory retrieval and impairs the ability of PrL neurons to dynamically distinguish between distinct phases of fear learning. Using transgenic and circuit-specific molecular targeting approaches, we demonstrate that unique patterns of activity- dependent gene transcription associated with brain-derived neurotrophic factor (BDNF) signaling within vHC- PrL projectors causally regulate activity in excitatory and inhibitory PrL neurons during context fear memory retrieval.

Conclusions: Together, our data show that activity-dependent BDNF release from molecularlydistinct vHC- PrL projection neurons modulates post-synaptic signaling in both inhibitory and excitatory PrL neurons, modifying activity in discrete populations of PrL neurons to suppress freezing during context fear memory retrieval.

\section{Keywords}

Context; Fear; Hippocampus; Prelimbic Cortex; Circuit; Gene Expression

\section{Introduction}

Aberrant prefrontal cortex (PFC) function is implicated in neuropsychiatric disorders, including generalized anxiety disorder (GAD)(1), and post-traumatic stress disorder (PTSD) (2), and patients with PTSD show altered regulation of fear(3) and deficits in emotional memory(4). Theories of emotional memory dysregulation in PTSD have centered on deficient context processing $(5,6)$, suggesting that patients with PTSD have core functional deficits in PFC circuits that are critical for relating emotional memories with the context in which they occur.

In rodents, the prelimbic (PrL) subdivision of the medial PFC (mPFC) critically contributes to fear memory expression during retrieval(7), while the infralimbic (IL) subdivision critically contributes to fear extinction(8). Recent evidence, however, suggests that the relationship between PrL function and fear behavior is more nuanced. For example, stimulating excitatory PrL-IL connections suppresses freezing during fear retrieval(9), and bulk calcium transients in the PrL predict decreases in freezing during extinction training(10), suggesting that the PrL can change how it represents freezing during memory formation, retrieval, and extinction. The PrL also receives input from the hippocampus (HC), which is critical for context-dependent fear expression(11) and also exhibits abnormal function in individuals with PTSD $(12,13)$. In rodents, the $\mathrm{HC}$ sends efferent projections to the PrL via neurons in the ventral CA1 subfield(14,15), suggesting that PrL-projecting neurons in the ventral HC (vHC-PrL projectors) may be a critical link between these two brain regions that have both been implicated in fear-related symptoms in neuropsychiatric disease. Can PrL neurons code for shifts in fear state during memory formation, context retrieval, and extinction? If so, how do inputs from the vHC affect this process at the circuit, cellular, and molecular levels?

We demonstrate that activation of monosynaptic ventral hippocampal (vHC) inputs to the PrL suppresses freezing during context fear memory retrieval by rendering PrL population activity more similar between context retrieval and extinction training. We further show that 
vHC-PrL projection neurons express transcripts that are implicated in diverse clinical gene sets, and that activation of these neurons induces unique patterns of activity-dependent gene transcription associated with brain-derived neurotrophic factor (BDNF) signaling in the mPFC. Finally, we find that selective expression of BDNF in vHC-PrL projection neurons is sufficient to rescue behavioral and molecular phenotypes associated with impaired HC-PFC function and attenuate exaggerated context fear memory expression in mice.

\section{Methods Subjects}

Male wild-type (w/t) C57B16/J and -e4 mice were group-housed (3-5 animals per cage) and maintained on a $12 \mathrm{~h}$ light/dark cycle in a temperature and humidity-controlled colony room. All animals had ad libitum access to food and water. Animals were 10-16 weeks of age, and weighed $25-35 \mathrm{~g}$ at time of surgery. All procedures were in accordance with the Institutional Animal Care and Use Committee of SoBran Biosciences Inc.

\section{Behavior}

For fear conditioning, animals were conditioned to associate a $30 \mathrm{~s}$ tone $(4000 \mathrm{~Hz})$ with a 2 s, $0.6 \mathrm{~mA}$ foot-shock. 4 tone-shock combinations were given, with the shock co-terminating with the last $2 \mathrm{~s}$ of each tone presentation. Tone-shock combinations were separated by $90 \mathrm{~s}$ intervals. $48 \mathrm{~h}$ later, mice were placed back into the conditioning chamber to measure retrieval of the conditioning context (in the absence of the tone) for $180 \mathrm{~s}$.

For full Methods, please see Supplemental Information.

\section{Results}

\section{Molecular characterization of vHC-PrL projection neurons.}

To isolate HA-tagged ribosome-mRNA complexes from vHC-PrL projectors, we injected a Cre-expressing retrograde virus (AAVrg-hSyn1-eBFP-Cre) into the PrL of RiboTag mice (Fig. 1a). We performed anti-HA immunohistochemistry in a subset of RiboTag mice, which revealed dense labeling restricted to the CA1 subfield of the vHC in the projector group, but widespread labeling in the control group (all Syn1-expressing vHC neurons) (Fig. 1b). We next immunoprecipitated (IP) ribosomes from HA-expressing neurons, and isolated RNA for sequencing. We used qPCR to confirm that virally-derived $C r e$ and $B f p$ were selectively expressed in IP samples from the projector group (Supp. Fig. 1a), further demonstrating projector-specific expression of the RiboTag allele. RNA-seq revealed that, compared to the control group, 644 genes were enriched in projectors, and 699 genes were depleted in projectors (Fig. 1c; Supp. Table 1), and gene ontology (GO) analysis showed that genes associated with learning, memory, synaptic plasticity, and stress signaling were enriched in vHC-PrL projectors (Supp. Fig. 1a; Supp. Table 2). We also found significant enrichment of our differentially-expressed genes among MESH term-defined clinical gene sets in the Harmonizome database(16), particularly among CNS disorders and traits. Many of these gene sets showed broad enrichment for diverse CNS (and non-CNS) disorder, among both projecting and non-projecting neurons even though the differentially expressed genes tested for enrichment were non-overlapping, suggesting broad neuronal enrichment. Secondary analyses assessed the specificity of these associations using a background of preferentially 
neuronally-expressed genes (see Methods), which showed expression of sets related to motor skills, attention-deficit hyperactivity disorder (ADHD), and substance-related disorders (Supp. Table 3).

\section{Stimulation of vHC-PrL projection neurons suppresses expression of context fear memories.}

We next injected a retrograde virus encoding a fluorescent reporter (AAVrg-CAG-tdTomato) into the PrL of wild-type (w/t) mice to label monosynaptic inputs, and performed c-Fos immunohistochemistry to identify tdTomato+ neurons in ventral CA1 that were activated during context fear retrieval in mice that were fear conditioned (shock group), versus controls (no shock group) (Fig. 1d). Mice in the shock group froze at significantly higher levels during both conditioning and context retrieval (Fig. 1e). The proportion of tdTomato+ neurons that co- expressed c-Fos (Fig. 1f) was significantly higher in the shock group, as compared to the no shock group (Fig. 1g), even as total number of c-Fos+ neurons did not significantly differ between groups (Supp. Fig. 1b). To causally link function in vHC-PrL projectors with context fear retrieval, we injected a retrograde virus expressing Crerecombinase (AAVrg-hSyn1-emBFP-Cre) into the PrL, along with a virus encoding for either Cre-dependent expression of the excitatory DREADD receptor hM3Dq (AAV5hSyn1-DIO-hM3Dq-mCherry) or the mCherry reporter alone (AAV5-hSyn1-DIO-mCherry) into the vHC of w/t mice, allowing us to selectively stimulate $\mathrm{vHC}$-PrL projectors prior to context retrieval. Mice in both groups were fear conditioned and administered clozapine-Noxide (CNO; $5 \mathrm{mg} / \mathrm{kg}$, i.p.) 45 minutes prior to context retrieval to engage the hM3Dq receptor (Fig. 1h). Synthetic activation of vHC-PrL projectors significantly decreased freezing during context retrieval (Fig. 1i), suggesting that vHC-PrL projectors regulate the suppression of context fear expression.

\section{Stimulation of vHC-PrL projectors inhibits flexible encoding of distinct fear memory stages in PrL neurons.}

We compared calcium dynamics within individual PrL neurons across distinct fear memory phases by injecting AAV1-hSyn1-GCaMP6f into the PrL and imaging calcium activity from 20-100 PrL neurons simultaneously across conditioning, context fear retrieval, and extinction training through an endoscopic lens with a miniature head-mounted microscope (57 \pm 12.65 neurons per animal, mean \pm s.e.m.; Fig. 2a). We reliably registered imaged neurons included in our analyses across all fear memory phases, allowing us to compare freezing-related activity in the same neuron as a function of conditioning (Fig. 2b). The majority of PrL neurons imaged were active across fear memory phases (Fig. 2c), and many PrL neurons dynamically represented freezing bouts, showing either increased or decreased calcium activity during freezing onset (Fig. 2d). For each fear memory phase, populations of PrL neurons exhibited a heterogeneous pattern of freezing-related activity (Supp. Fig. 2). However, all imaged PrL neurons changed their activity patterns during freezing bouts as a function of fear memory phase (Fig. 2e); for example, a neuron that had increased calcium activity during fear retrieval freezing bouts could have decreased calcium activity during extinction training freezing bouts, and vice versa, with no stereotyped pattern detectable. 
We next injected a combination of viruses to induce expression of either the excitatory hM3Dq receptor or an mCherry control selectively in vHC-PrL projectors, and administered $\mathrm{CNO}$ prior to context retrieval while simultaneously imaging calcium dynamics in the PrL during fear retrieval and within-session extinction. Unilateral activation of vHC-PrL projectors did not alter the number of freezing bouts during any fear memory phase (Supp Fig. 2a), but did impact patterns of freezing-related activity in ipsilateral PrL neurons (Supp. Fig. 2c). To quantify variability of PrL neuron responses across fear memory phases at the population level, we used linear mixed-effects modeling, which showed that mean freezingrelated calcium activity increased from context retrieval to early extinction training in mCherry controls, and that this effect was blunted in hM3Dq animals (Fig. 2f), indicating that projector activation renders the PrL less able to flexibly represent distinct fear memory phases during freezing.

Using principal components analysis (PCA), we further found that clusters of PrL ensemble activity were highly separated along the first two principal components as a function of fear memory phase (Fig. 2g). If individual neurons in the PrL change their freezing response as a function of fear learning and memory, then it should be possible to predict fear memory phase from freezing-related PrL population activity. To test this, we trained a support vector machine (SVM) classifier to distinguish between early and late conditioning, or context retrieval and early extinction training based on PrL population calcium activity. We found that decoding accuracy of the classifier was higher than chance (shuffled data) in both groups when distinguishing between early and late conditioning, but fell to chance levels in hM3Dq animals when choosing between context retrieval and early extinction (Fig. 2h). Taken together, our data suggest that vHC inputs to the PrL suppress context fear retrieval by rendering PrL population states more rigid between retrieval and extinction training.

\section{vHC-PrL projector activation induces distinct activity-dependent gene expression profiles in the mPFC.}

To investigate how vHC-PrL projector stimulation impacts PrL activity at the molecular level, we expressed hM3Dq or an mCherry control selectively in vHC-PrL projectors and administered $\mathrm{CNO}$ to assess effects of projector activation on gene expression in the $\mathrm{mPFC}$ (Fig. 3a). We used qPCR to confirm that activation of vHC-PrL projectors induced immediate early gene (IEG) expression in bulk mPFC tissue, and found significant enrichment ( 10-30 fold increases) of several IEGs in the mPFC of the CNO+hM3Dq group, as compared to both control groups (Fig. 3b).

We next performed RNA-seq on isolated mPFC RNA from CNO+hM3Dq and saline $+\mathrm{hM} 3 \mathrm{Dq}$ groups, and identified 1,155 genes that were differentially expressed between the two groups at FDR < 5\% (Fig. 3c; Supp. Table 4). We next performed GO enrichment analysis on the subset of 602 genes that were significantly enriched in the CNO+hM3Dq group, and found that vHC-PrL stimulation induced mRNAs for genes that encode proteins critically involved in learning, memory, and cognition (Fig. 3d; Supp. Table 5). On further examination of our gene set, we noted that several genes in the brain-derived neurotrophic factor (BDNF)-TrkB signaling pathway were significantly enriched (Fig. 3e). This indicates that projector activation affects BDNF- TrkB signaling in the mPFC during context fear 
memory retrieval, which is in line with previous literature demonstrating a critical role for BDNF signaling in hippocampal-prefrontal circuitry and fear behavior $(17,18)$. To more conclusively link BDNF function with differentially expressed genes in our data set, we compared fold changes in significantly regulated genes from our data with a data set of significantly regulated genes following application of BDNF to cultured cortical neurons(19). We found highly significant directional overlap of fold changes in differentially expressed genes between the two data sets (Fig. 3f), further implicating BDNF-TrkB signaling as a key regulator of function in the vHC-mPFC circuit.

\section{Context fear retrieval induces distinct molecular phenotypes in the PrL of mutant mice with decreased activity-dependent BDNF signaling.}

To relate BDNF-TrkB signaling with PrL function during fear behavior, we examined cell type-specific patterns of activation following context fear retrieval in mutant mice with disruption of activity-dependent BDNF production (-e4 mice). In these mice, knock-in of an eGFP-STOP cassette to the $B d n f$ exon IV locus causes disruption of BDNF production from promoter IV, while leaving production from other Bdnf promoters intact(20) (Fig. 4a). We have previously shown that, in contrast to mutant mice with disruption of BDNF from other promoters, -e4 mice have exaggerated freezing levels during fear retrieval that are concomitant with impairments in hippocampal-prefrontal synchrony(21). These data, along with other literature demonstrating modifications in promoter IV-derived $B d n f$ expression following fear behavior $(22,23)$ led us to conclude that $B d n f$ transcripts derived from promoter IV were the most likely source of hippocampal-prefrontal BDNF influencing context fear expression. No significant difference in freezing was observed between genotypes during conditioning, but -e4 mice froze at significantly higher levels than w/t controls during context retrieval (Fig. 4c). Ninety minutes following context retrieval, we sacrificed mice and extracted brains for analysis of fear-related patterns of gene expression in the PrL and IL. We used single- molecule in situ hybridization to identify excitatory neurons (Slc17a7 probe) recruited during context fear retrieval (Fos probe) that could be directly modulated by BDNF (Ntrk2 probe) (Fig. 4d). We found that a higher proportion of excitatory cells expressing Ntrk2 (SIc17a7+/Ntrk2+) in the PrL co-expressed Fos in -e4 mice, and that the same population of Slc17a7+/Ntrk2+ neurons in the IL, which is necessary for fear extinction, co- expressed Fos to a significantly lesser degree in -e4 animals following context fear retrieval (Fig. 4e). We repeated our experiment with a probe for Gad1 to assess the degree to which inhibitory neurons in the PrL and IL were recruited during context fear retrieval in both genotypes (Fig. 4f). We found an opposing phenotype in the PrL of -e4 mice, such that a lower proportion of Gad1+/Ntrk2+ neurons co-expressed Fos in the PrL of -e4 mice compared to w/t mice. These data support the hypothesis that local excitatory-inhibitory micro-circuits in the PrL regulate context fear memory retrieval, and that these micro-circuits are influenced by BDNF- modulated synaptic input, possibly from the vHC (Fig. 4g).

\section{BDNF-TrkB signaling in vHC-PrL projectors impacts their physiology and recruitment during context fear retrieval.}

We again used single-molecule in situ hybridization to investigate cell-type specific expression of $t d T$ Tomato (vHC-PrL projectors), Fos (retrieval-activated cells), and exon IV- 
containing $B d n f$ in ventral $\mathrm{CA} 1$ of w/t and -e4 mice following context fear retrieval (Fig. 5a). We compared levels of Fos expression in projectors between w/t and -e4 mice, and found no significant difference in proportion of projectors co-expressing Fos transcripts or number of Fos transcripts per projector between genotypes (Supp. Fig. 3a), suggesting that effects of projector activation on downstream brain regions, but not projector activation per se, contributes to context fear memory retrieval. Exon IV-containing $B d n f$ transcripts were significantly enriched in projectors (Supp. Fig. 3b), with Fos-expressing projectors containing the highest number of $B d n f$ transcripts (Fig. 5b; Supp. Fig. 3c). While BDNF is not translated from exon IV Bdnf-Gfp transcripts in -e4 mice, Gfp-containing transcripts can be quantified as a readout of transcriptional activity at promoter IV. We found that projectors activated during context fear memory retrieval were highly enriched for exon IV-containing transcripts in both w/t and -e4. However, a relatively higher percentage of tdTomato+/Fost, tdTomato+/Fos-, and tdTomato-/Fos+ neurons co-expressed Bdnf-Gfp transcripts in -e4 mice following context fear retrieval, as compared to $\mathrm{w} / \mathrm{t}$ mice (Fig. $5 \mathrm{c}$ ). Since it is possible that differences in transcript expression between genotypes, or specificity of the $B d n f$ probe in the -e4 mutants, could drive observed differences in Bdnf levels in the vHC, we repeated our experiment with a probe for $G f p$, and found results that mirrored those observed with $B d n f$ (Supp. Fig. 3d), indicating that transcriptional activity at promoter IV is indeed increased in -e4 mice following context fear retrieval.

The observed increase in transcriptional activity at Bdnf promoter IV in -e4 vHC-PrL projectors could arise from altered synaptic physiology or excitability of these cells. To test this, we injected AAVrg-CAG-tdTomato into the PrL to label projectors, and cut acute slices of the vHC to record from tdTomato+ cells ex vivo (Fig. 5f). We found that -e4 projectors were similar to w/t projectors in terms of input/output firing rate curves (Fig. $5 \mathrm{~g}$ ), maximum number of action potentials in response to current injections (Supp. Fig. 4a), $\mathrm{Na}^{+}$current amplitude (Supp. Fig. 4b), and threshold (Supp. Fig. 4c). Neither the frequency nor amplitude of excitatory PSCs (ePSCs) onto projectors significantly differed between genotypes (Fig. 5h); however, the frequency of inhibitory PSCs (iPSCs) onto projectors was significantly lower in -e4 mice, even as the amplitude of iPSCs did not significantly differ between $\mathrm{w} / \mathrm{t}$ and -e4 mice (Fig. 5i). - e4 projectors had a correspondingly more depolarized resting membrane potential (RMP) as compared to w/t projectors (Fig. 5j), in the absence of significant differences in membrane capacitance (Supp. Fig. 4d) or resistance (Supp. Fig. 4e). Taken together, these results suggest that, even though w/t and -e4 projectors generate action potentials at the same rate, action potential generation in - 4 projectors is potentially impacted by altered inhibitory synaptic input.

Given that dendritic release of BDNF from CA1 neurons can trigger autocrine BDNF-TrkB signaling that drives structural and functional plasticity(24), we also assessed whether vHCPrL projectors are themselves targets of BDNF signaling by using single-molecule in situ hybridization with probes for Fos, tdTomato, and Ntrk2 (Fig. 5d). Projectors were significantly enriched for Ntrk2 mRNA (Supp. Fig. 3e), and projectors activated during context fear retrieval (tdTomato+/Fos+) co-expressed Ntrk 2 at the highest levels, with no difference in Ntrk 2 co-expression between -e4 and w/t mice (Fig. 5e). Taken together, these results demonstrate that $\mathrm{vHC}-\mathrm{PrL}$ projectors engage distinct plasticity cascades in response to behavior, with projectors being enriched for genes associated with BDNF-TrkB signaling. 


\section{Activation of vHC-PrL projectors produces opposite effects on context fear retrieval and freezing- related PrL activity in -e4 mice.}

Our data thus far suggest that less pre-synaptic BDNF release from vHC-PrL projectors and blunted TrkB activation in the PrL may contribute to increases in context fear expression during retrieval in -e4 animals. To test this hypothesis, we virally introduced either the excitatory DREADD receptor hM3Dq or a control reporter (mCherry) into vHC-PrL projectors of -e4 mice, and injected CNO $(5 \mathrm{mg} / \mathrm{kg}$, i.p.) $45 \mathrm{~m}$ prior to context fear retrieval (Fig. 6a). In contrast to the suppression of freezing that was observed during context retrieval following projector activation in $\mathrm{w} / \mathrm{t}$ mice, projector activation in -e4 mice significantly increased freezing during context retrieval (Fig. 6b). We further compared calcium activity in the PrL of mCherry controls in which projectors were left un-stimulated to hM3Dq mice in which projectors were stimulated prior to context retrieval (Fig. 6c \& 6d). In mCherry -e4 control mice, individual PrL neurons tended to maintain a consistent freezing-related activity profile across fear memory phases (Fig. 6e), suggesting that abnormal context retrieval in -e4 mice may be due to abnormal population representations between fear epochs. We found that application of CNO prior to retrieval in hM3Dq mice led to increased freezing-related $\Delta \mathrm{f} / \mathrm{f}$ during early extinction training as compared to retrieval; this effect was not observed in mCherry animals (Fig. 6f). A SVM classifier discriminated between early and late conditioning freezing bouts at levels above chance for both groups, but failed to distinguish between context retrieval and extinction training only in the mCherry group (Fig. 6g), demonstrating that decreased activity-dependent BDNF production impedes the ability of PrL neurons to dynamically represent distinct fear memory phases, and that stimulation of vHC-PrL projectors in the absence of promoter IV-derived BDNF paradoxically increases freezing during context fear retrieval.

\section{Expression of BDNF selectively in vHC-PrL projectors reverses behavioral and molecular phenotypes during context fear retrieval in -e4 mice.}

If decreased BDNF in vHC-PrL projectors increases context fear expression, we reasoned that exogenous expression of BDNF in vHC-PrL projectors would decrease exaggerated fear expression in -e4 animals. To test this, we injected AAVrg-hSyn1-emBFP-Cre into the PrL and AAV8-CAG-FLEX-BDNF:HA into the vHC for the Cre-dependent expression of a BDNF expression cassette in our experimental group, and injected AAV8-hSyn1-DIOmCherry into the $\mathrm{vHC}$ in our control group for Cre-dependent expression of the mCherry reporter (Fig. 7a). In our construct, BDNF is fused to an HA tag, which was readily detectable in vHC-PrL projectors with anti-HA immunohistochemistry (Fig. 7b). We found that selective BDNF expression in projectors (BDNF:HA group) decreased freezing during retrieval (Fig. 7c), supporting our hypothesis that BDNF synthesis in projectors is sufficient to suppress context fear.

Using single-molecule in situ hybridization, we found that the proportion of Slc17a7+l Ntrk2+ neurons that co- expressed fear-induced Fos was significantly lower in the PrL of BDNF:HA mice. We did not find any significant difference in recruitment of Slc17a7+/ $N t k 2+$ neurons between groups in the IL, indicating that changes in IL function during context fear retrieval in -e4 mice are not driven by differences in PrL signaling (Fig. 7d). Unexpectedly, we did find that Fos co-expression in Gad1+/Ntrk2+ neurons was 
significantly lower in the IL of BDNF:HA mice (Fig. 7e), suggesting that decreased recruitment of PrL excitatory neurons during context fear suppression may influence inhibitory signaling in the IL. Given that we did not observe an opposite phenotype in -e4 mice during context fear retrieval (i.e., increased recruitment of inhibitory neurons in the IL), this suggests that parallel pathways to the PrL and IL likely contribute to increased fear expression in mutant animals.

\section{Discussion}

\section{vHC-PrL Projectors Alter PrL Population Dynamics to Suppress Context Fear Memory Retrieval}

Our findings demonstrate a novel role for direct ventral hippocampal inputs to the PrL in suppression of freezing during retrieval of a fear-associated context. The $\mathrm{vHC}$ is known to be necessary for both tone $(25,26)$ and context $(11)$ fear retrieval, but the efferent projections within the $\mathrm{vHC}$ that mediate these processes are not known. Indeed, we found that the total number of c-Fos+ neurons in the vHC following re-exposure to the conditioning chamber did not significantly differ between conditioned and non-conditioned animals, suggesting that environmental novelty is a strong driver of c-Fos expression in this brain region $(27,28)$. We did, however, find a difference in proportion of vHC-PrL projectors that co-expressed cFos between these groups, suggesting that spatially-distinct vHC circuits selectively participate in different aspects of fear behavior. Early studies showed that vHC inactivation increases firing rates in PrL neurons during tone presentations in conditioned animals(29), suggesting that $\mathrm{vHC}$ outputs to the PrL might mediate retrieval of tone-shock associations. The use of muscimol to inactivate vHC neurons in that study, however, precluded a conclusion about how PrL-projecting neurons in the vHC participate in this process. Indeed, a recent study in which DREADD receptors were expressed exclusively in vHC-PrL projectors revealed that neither activation nor inactivation of these neurons affects freezing during tone retrieval in conditioned mice(30). These projections have also been shown to affect spatial working memory(31) and anxiety(32) in mice, suggesting that this pathway supports both the emotional and memory-related aspects of context fear expression. Inactivation of the PrL decreases freezing during both tone(7) and context(33) retrieval, while micro-stimulation of the PrL increases freezing during tone presentations in conditioned animals(34). In contrast, local inactivation of the IL does not affect freezing during context retrieval, but does impair extinction learning and extinction retrieval(8). A host of other studies have demonstrated that other brain regions, including the amygdala $(35,36,37)$, play a key role in both fear expression and extinction, suggesting that vHC-PrL circuitry is not the only pathway that is critically involved in context fear expression. Rather, it is likely that vHC-PrL projectors are one important pathway within a network of neural circuits that work together to regulate fear expression.

\section{Molecular Signatures in the vHC-PrL Pathway}

We demonstrate that $\mathrm{vHC}-\mathrm{PrL}$ projectors are molecularly distinct from other vHC neurons (Fig. 1c), including those in CA1 that do not project to the PrL (Fig. 5c \& Fig. 5e). These results indicate that genetically distinct sub-populations in the $\mathrm{vHC}(38)$ may send axons that synapse onto similar downstream brain areas. We further show that vHC-PrL projectors are 
enriched for gene sets that are implicated in a number of diseases, suggesting that molecular profiles of projection-specific populations in rodents could be "mapped on" to risk gene sets in human neuropsychiatric disorders. Synthetic activation of $\mathrm{vHC}-\mathrm{PrL}$ projectors induced activity- dependent gene programs in the $\mathrm{mPFC}$, further demonstrating that the $\mathrm{mPFC}$ transcriptome can be dynamically affected by activation of anatomically-distinct upstream inputs. Activity-dependent gene expression profiles differ according to an animal's experience in a number of different brain areas, including the $\operatorname{mPFC}(39)$, indicating that distinct events (i.e., fear memory retrieval vs. social interaction) can have unique experiencedependent "molecular signatures"(40). The identification of transcriptomic signatures related to activation of spatially-localized neural circuits could compliment these findings by linking these circuits with defined behaviors at the molecular level. These findings may also have implications for the identification of unique biomarkers associated with neuropsychiatric disorders, many of which are associated with aberrant PFC function(41). To definitively conclude that activation of hippocampal inputs causes unique patterns of gene transcription in the $\mathrm{mPFC}$, future experiments should measure gene expression in the mPFC following stimulation of other prefrontal afferents (e.g., amygdala, brainstem).

\section{Activity-Dependent BDNF Signaling Alters vHC-PrL Function During Context Fear Memory Retrieval}

We demonstrate that fold-changes in our RNA-seq data set are correlated with fold-changes in another RNA- seq data set of cultured neurons following application of $\operatorname{BDNF}(19)$, indicating that stimulation of vHC-PrL projectors induces BDNF-TrkB signaling within the mPFC. Previous work has demonstrated relationships between hippocampal-prefrontal function, BDNF, and fear behavior $(17,18)$, including work from our laboratory showing that mice with decreased production of activity-dependent BDNF have deficits in fear extinction that co-occur with impaired hippocampal-prefrontal oscillatory synchrony(21). Transcription from promoter IV of the $B d n f$ gene in the dorsal hippocampus is correlated with fear memory consolidation(22) and epigenetic modification of promoter IV in the IL is linked with fear extinction $(23,42)$, findings that suggest that BDNF production from promoter IV may contribute to the mechanism underlying fear behavior(43). Our lab recently demonstrated that synthetic excitation of cells in the ventral dentate gyrus expressing promoter IV-derived $B d n f$ transcripts increased freezing during context fear retrieval and concomitantly attenuated vHC-PrL oscillatory synchrony(44). The current data extend this finding by showing that promoter IV-derived $B d n f$ is enriched in a projection-specific population of $\mathrm{vHC}$ neurons and that global disruption of promoter IV-derived BDNF directly impacts function in this projector population.

Paradoxically, in contrast to w/t animals, stimulation of -e4 vHC-PrL projectors increased freezing during context fear retrieval, suggesting that synthesis and release of activitydependent BDNF from projectors, and not activation of projectors per se, drives decreased contextually-driven fear expression. We chose to stimulate projectors in order to understand how these neurons might drive fear behavior in mice with different genotypes. We would expect, however, that inhibition of these neurons would produce opposite effects on behavior - e.g., projector inhibition (and hence, suppression of BDNF release from these neurons) in w/t mice should increase fear expression, and projector inhibition in -e4 mice should 
decrease fear expression. Exogenous expression of BDNF protein selectively in vHC-PrL projectors reversed behavioral and molecular phenotypes in the PrL of -e4 mice during context fear retrieval. These data demonstrate that genetic background can critically bias circuit function, which must be taken into account for circuit-targeting strategies in symptoms of neuropsychiatric disease; for example, in individuals with functional singlenucleotide polymorphisms (SNPs) in the $B D N F$ gene, which can affect activity-dependent BDNF secretion(45) and fear behavior(46).

Relating prefrontal function with retrieval of a fear-associated context will be important for understanding how contextually-specific fear responses are dysregulated in $\operatorname{PTSD}(5,6)$; the rodent PrL is thought, in part, to be functionally homologous to the primate anterior cingulate cortex (47), which functions aberrantly during emotional responding in PTSD patients(48).

\section{Supplementary Material}

Refer to Web version on PubMed Central for supplementary material.

\section{Acknowledgements}

The authors wish to thank Richard de los Santos Abreu, Madhavi Tippani, Danisha Gallop, Leon Lin, and Martha Kimos for technical assistance. The authors additionally wish to thank Daniel Weinberger for invaluable comments on earlier versions of the manuscript. This project was supported by the Lieber Institute for Brain Development and by National Institutes of Health awards to KM (R01MH105592) and HH (F32MH121052).

\section{References}

1. Greenberg T, Carlson JM, Cha J, Hajcak G \& Mujica-Parodi LR Ventromedial prefrontal cortex reactivity is altered in generalized anxiety disorder during fear generalization. Depress. Anxiety 30, 242-250 (2013). [PubMed: 23139148]

2. Zubieta JK, Chinitz JA, Lombardi U, Fig LM, Cameron OG, \& Liberzon I. Medial frontal cortex involvement in PTSD symptoms: a SPECT study. J. Psychiatr. Res. 33, 259-264 (1999). [PubMed: 10367992]

3. Lanius Ruth A., Frewen Paul A., Vermetten E, \& Yehuda R. Fear conditioning and early life vulnerabilities: Two distinct pathways of emotional dysregulation and brain dysfunction in PTSD. Eur. J. Psychotraum, 1: 5467 (2010).

4. Parsons RG \& Ressler KJ Implications of memory modulation for post-traumatic stress and fear disorders. Nat. Neurosci. 16, 146-153 (2013). [PubMed: 23354388]

5. Liberzon I, \& Abelson JL Context processing and the neurobiology of post-traumatic stress disorder. Neuron, 92, 14-30 (2016). [PubMed: 27710783]

6. Maren S, Phan KL, \& Liberzon I. The contextual brain: Implications for fear conditioning, extinction and psychopathology. Nat. Rev. Neurosci, 14, 417-428 (2013). [PubMed: 23635870]

7. Corcoran KA \& Quirk GJ Activity in prelimbic cortex is necessary for the expression of learned, but not innate, fears. J. Neurosci. 27, 840-844 (2007). [PubMed: 17251424]

8. Morgan MA, Romanski LM \& LeDoux JE Extinction of emotional learning: contribution of medial prefrontal cortex. Neurosci. Lett. 163, 109-113 (1993). [PubMed: 8295722]

9. Marek R, Xu L, Sullivan RKP \& Sah P. Excitatory connections between the prelimbic and infralimbic medial prefrontal cortex show a role for the prelimbic cortex in fear extinction. Nat. Neurosci. 21, 654-658 (2018). [PubMed: 29686260]

10. Giza JI, Kim J, Meyer HC, Anastasia A, Dincheva I, Zheng CI, et al. The BDNF val66met prodomain disassembles dendritic spines altering fear extinction circuitry and behavior. Neuron 99, 163-178.e6 (2018). [PubMed: 29909994] 
11. Hobin JA, Ji J \& Maren S. Ventral hippocampal muscimol disrupts context-specific fear memory retrieval after extinction in rats. Hippocampus 16, 174-182 (2006). [PubMed: 16358312]

12. Aster RS, St. Germain SA, Tolin D, Ford J, Russell D, \& Stevens M. Hippocampus function predicts severity of post-traumatic stress disorder. CyberPsych. \& Behav, 9: 234-240 (2006)

13. Hayes JP, LaBar KS, McCarthy G, Selgrade E, Nasser J, Dolcos F, et al. Reduced hippocampal and amygdala activity predicts memory distortions for trauma reminders in combat-related PTSD. J. Psych. Res, 45: 660-669 (2011).

14. Swanson LW A direct projection from Ammon's horn to prefrontal cortex in the rat. Brain Res. 217, 150-154 (1981). [PubMed: 7260612]

15. Jay TM, Burette F \& Laroche S. Plasticity of the hippocampal-prefrontal cortex synapses. J Physiol Paris 90, 361-366 (1996). [PubMed: 9089514]

16. Rouillard AD, Gundersen GW, Fernandez NF, Wang Z, Monteiro CD, McDermott MG, et al. The harmonizome: A collection of processed datasets gathered to serve and mine knowledge about genes and proteins. Database, doi: 10.1093/database/baw100.

17. Peters J, Dieppa-Perea LM, Melendez LM, \& Quirk GJ Induction of fear extinction with hippocampal-infralimbic BDNF. Science, 39, 2161-2169 (2010).

18. Rosas-Vidal LE, Do-Monte FH, Sotres-Bayon F \& Quirk GJ Hippocampal--prefrontal BDNF and memory for fear extinction. Neuropsychopharmacology 39, 2161-2169 (2014). [PubMed: 24625752]

19. Hixson KM, Cogswell M, Brooks-Kayal AR \& Russek SJ Transcriptomic analysis of the BDNFinduced JAK/STAT pathway in neurons: a window into epilepsy-associated gene expression. BioRxiv (2019). doi:10.1101/577627

20. Maynard KR, Hill JL, Calcaterra NE, Palko ME, Kardian A, Paredes D, et al. Functional role of bdnf production from unique promoters in aggression and serotonin signaling. Neuropsychopharmacology 41, 1943-1955 (2016). [PubMed: 26585288]

21. Hill JL, Hardy NF, Jimenez DV, Maynard KR, Kardian AS, Pollock CJ, et al. Loss of promoter IVdriven BDNF expression impacts oscillatory activity during sleep, sensory information processing and fear regulation. Transl. Psychiatry 6, e873 (2016). [PubMed: 27552586]

22. Lubin FD, Roth TL \& Sweatt JD Epigenetic regulation of BDNF gene transcription in the consolidation of fear memory. J. Neurosci. 28, 10576-10586 (2008). [PubMed: 18923034]

23. Bredy TW, Wu H, Crego C, Zellhoefer J, Sun YE, \& Barad M. Histone modifications around individual BDNF gene promoters in prefrontal cortex are associated with extinction of conditioned fear. Learn. Mem. 14, 268-276 (2007). [PubMed: 17522015]

24. Harward SC, Hedrick NG, Hall CE, Parra-Bueno P, Milner TA, Pan E, et al. Autocrine BDNFTrkB signalling within a single dendritic spine. Nature 538, 99-103 (2016). [PubMed: 27680698]

25. Bast T, Zhang W-N \& Feldon J. The ventral hippocampus and fear conditioning in rats. Exp. Brain Res. 139, 39-52 (2001). [PubMed: 11482842]

26. Kjelstrup KG, Tuvnes FA, Steffenach HA, Murison R, Moser EI, \& Moser MB Reduced fear expression after lesions of the ventral hippocampus. Proc. Natl. Acad. Sci. USA 99, 10825-10830 (2002). [PubMed: 12149439]

27. Asok A, Schreiber WB, Jablonski SA, Rosen JB, \& Stanton ME Egr-1 increases in the prefrontal cortex following training in the context preexposure facilitation effect (CPFE) paradigm. Neurobiol. Learn. Mem. 106: 145-153 (2013). [PubMed: 23973447]

28. Wang ME, Fraize NP, Yin L, Yuan RK, Petsagourakis D, Wann EG, et al. Differential roles of the dorsal and ventral hippocampus in predator odor contextual fear conditioning. Hippocampus, 23: 451-466 (2013). [PubMed: 23460388]

29. Sotres-Bayon F, Sierra-Mercado D, Pardilla-Delgado E \& Quirk GJ Gating of fear in prelimbic cortex by hippocampal and amygdala inputs. Neuron 76, 804-812 (2012). [PubMed: 23177964]

30. Vasquez JH, Leong KC, Gagliardi CM, Harland B, Apicella AJ, \& Muzzio IA Pathway specific activation of ventral hippocampal cells projecting to the prelimbic cortex diminishes fear renewal. Neurobiol. Learn. Mem. 161, 63-71 (2019). [PubMed: 30898692]

31. Spellman T, Rigotti M, Ahmari SE, Fusi S, Gogos JA, \& Gordon JA Hippocampal-prefrontal input supports spatial encoding in working memory. Nature, 522: 309-314 (2015). [PubMed: 26053122] 
32. Padilla-Coreano N, Bolkan SS, Pierce GM, Blackman DR, Hardin WD, Garcia-Garcia AL, et al. Direct ventral hippocampal-prefrontal input is required for anxiety-related neural activity and behavior. Neuron, 89: 857-866 (2016). [PubMed: 26853301]

33. Kim EJ, Kim N, Kim HT \& Choi J-S The prelimbic cortex is critical for context-dependent fear expression. Front. Behav. Neurosci. 7, 73 (2013). [PubMed: 23801949]

34. Vidal-Gonzalez I, Vidal-Gonzalez B, Rauch SL \& Quirk GJ Microstimulation reveals opposing influences of prelimbic and infralimbic cortex on the expression of conditioned fear. Learn. Mem. 13, 728-733 (2006). [PubMed: 17142302]

35. Chudasama Y, Izquierdo A, \& Murray EA Distinct contributions of the amygdala and hippocampus to fear expression. Eur. J. Neurosci, 30: 2327-2337 (2009). [PubMed: 20092575]

36. Sierra-Mercado D, Padilla-Coreano N, \& Quirk GJ Dissociable roles of prelimbic and infralimbic cortices, ventral hippocampus, and basolateral amygdala in the expression and extinction of conditioned fear. Neuropsychopharm, 36: 529-538 (2011).

37. Pare D \& Duvarci S. Amygdala microcircuits mediating fear expression and extinction. Curr. Opin. Neurobiol, 22: 717-723 (2012). [PubMed: 22424846]

38. Cembrowski MS, Bachman JL, Wang L, Sugino K, Shields BC, \& Spruston N. Spatial geneexpression gradients underlie prominent heterogeneity of CA1 pyramidal neurons. Neuron 89 , 351-368 (2016). [PubMed: 26777276]

39. Mukherjee D, Ignatowska-Jankowska BM, Itskovits E, Gonzales BJ, Turm H, Izakson L, et al. Salient experiences are represented by unique transcriptional signatures in the mouse brain. eLife, 7: doi: 10.7554/eLife.31220.

40. Sagar V \& Kahnt T. Genetic signatures of memories. Elife 7, (2018).

41. Tekin S \& Cummings JL Frontal-subcortical neuronal circuits and clinical neuropsychiatry: an update. J. Psychosom. Res. 53, 647-654 (2002). [PubMed: 12169339]

42. Li X, Zhao Q, Wei W, Lin Q, Magnan C, Emami MR, et al. The DNA modification N6-methyl-2'deoxyadenosine (m6dA) drives activity-induced gene expression and is required for fear extinction. Nat. Neurosci. 22, 534-544 (2019). [PubMed: 30778148]

43. Hill JL \& Martinowich K. Activity-dependent signaling: influence on plasticity in circuits controlling fear-related behavior. Curr. Opin. Neurobiol. 36, 59-65 (2016). [PubMed: 26485574]

44. Hallock HL, Quillian HM, Mai Y, Maynard KR, Hill JL, \& Martinowich K. Manipulation of a genetically and spatially defined sub-population of BDNF-expressing neurons potentiates learned fear and decreases hippocampal-prefrontal synchrony in mice. Neuropsychopharmacology (2019). doi:10.1038/s41386-019-0429-1

45. Egan MF, Kojima M, Callicott JH, Goldberg TE, Kolachana BS, Bertolino A, et al. The BDNF val66met polymorphism affects activity-dependent secretion of BDNF and human memory and hippocampal function. Cell, 112, 257-269 (2003). [PubMed: 12553913]

46. Solimon F, Glatt CE, Bath KG, Levita L, Jones RM, Pattwell SS, et al. A genetic variant BDNF polymorphism alters extinction learning in both mouse and human. Science, 327, 863-866 (2010). [PubMed: 20075215]

47. Hoover WB, \& Vertes RP Anatomical analysis of afferent projections to the medial prefrontal cortex in the rat. Brain Struct. Func, 212: 149-179 (2007).

48. Lanius RA, Williamson PC, Boksman K, Densmore M, Gupta M, Neufeld RWJ, et al. Brain activation during script-driven imagery induced dissociative responses in PTSD: a functional magnetic resonance imaging investigation. Biol. Psych, 52: 305-311 (2002).: 
TRAP-mediated RNA-seq in vHC-PrL projectors

A

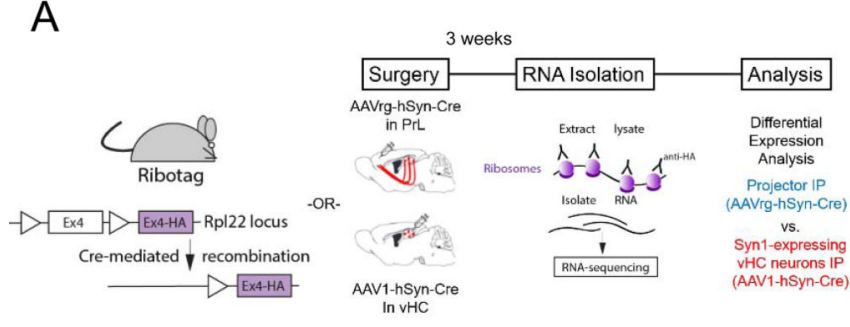

B

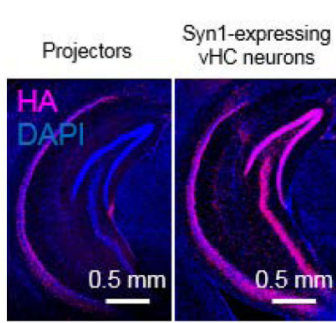

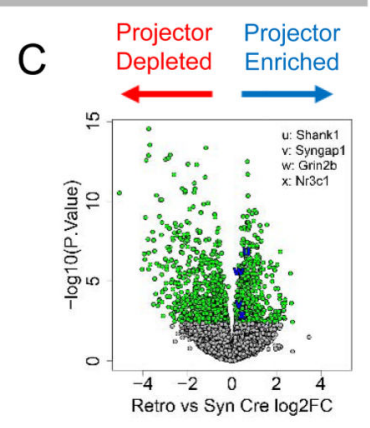

Recruitment of vHC-PrL projectors during context retrieval

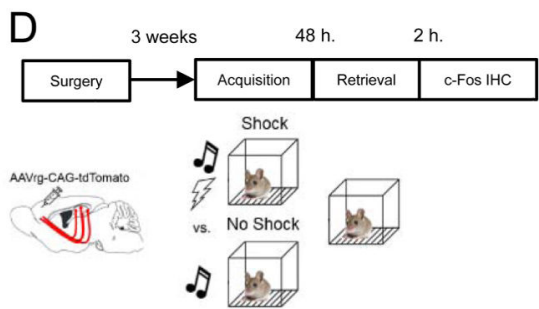

$\mathrm{F}$

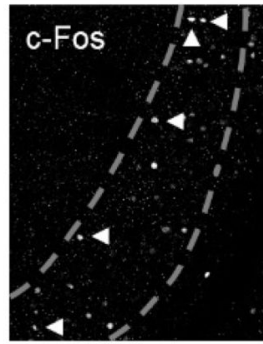

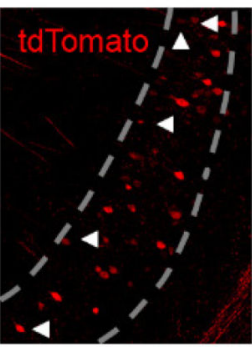

$\mathrm{E}$

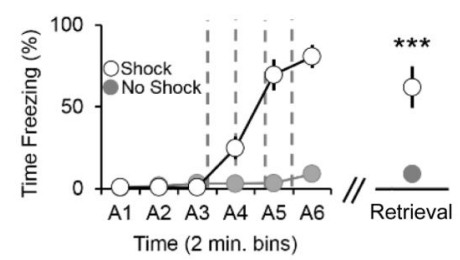

G

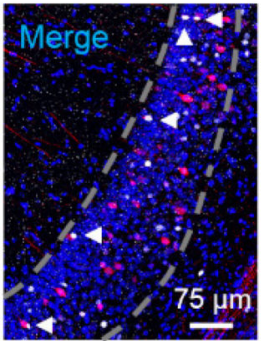

Activation of projectors prior to context retrieval

$\mathrm{H}$

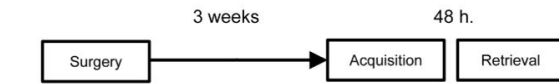

AAVrg-hSyn-Cre AAV5-hSyn-
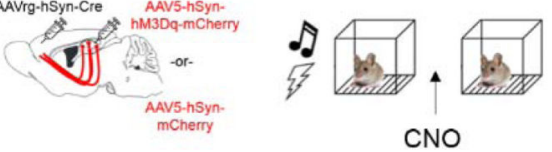

I
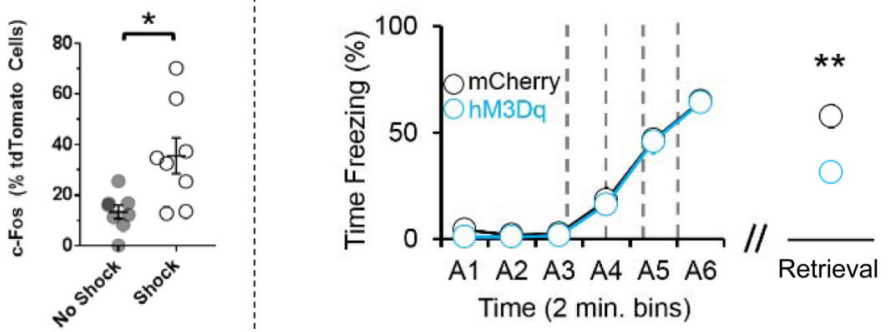

Figure 1.

Activation of molecularly-distinct vHC-PrL projectors suppresses context fear memory retrieval. (A) Schematic of timeline and experimental design for RNA-sequencing of projectors vs. all Syn1-expressing vHC neurons. (B) HA protein expression in the vHC of Rpl22 ${ }^{\mathrm{HA}}$ animals injected with AAVrg-hSyn1-emBFP-Cre in the PrL (left panel), and AAV1-hSyn1-emBFP-Cre in the vHC (right panel). (C) Volcano plot showing significantly enriched genes in projectors (green dots, right of 0 on x-axis) and significantly enriched genes in Syn1-expressing vHC neurons (green dots, left of 0 on x-axis). Shank1 log2(FC) = 0.65 , adjusted $p=0.0000187$, Syngap $1 \log 2 \mathrm{FC}=0.31$, adjusted $p=0.007$, Grin $2 b \log 2 \mathrm{FC}=$ 0.3 , adjusted $p=0.0002, N r 3 c 1 \log 2 \mathrm{FC}=0.45$, adjusted $p=0.024$. (D) Schematic of timeline and experimental design for identification of projectors recruited during context fear retrieval. (E) Mice that received a shock during conditioning froze at significantly higher levels than mice that did not receive a shock during both conditioning $(F(5,30)=$ 84.26, $p<0.0001$, mixed-factorial ANOVA) and context retrieval $(t(6)=7.075, p=0.0004$, unpaired t-test). (F) Example confocal z-projections from ventral CA1 showing tdTomato and c-Fos co-expression. $(G)$ A significantly larger proportion of projectors (tdTomato+ neurons) in ventral CA1 co-express context retrieval-induced c-Fos in shocked mice vs. nonshocked mice $(t(14)=2.933, p=0.01$, unpaired $\mathrm{t}$-test; $\mathrm{n}$ mice $=4$ per group, $\mathrm{n}$ images $=2$ 
per mouse). (H) Schematic of timeline and experimental design for excitation of vHC-PrL projectors prior to context fear retrieval. (I) Synthetic excitation of projectors prior to context retrieval significantly reduces freezing relative to viral (mCherry) controls $(t(26)=2.917, p$ $=0.007$, unpaired $\mathrm{t}$-test; $\mathrm{n}$ mice $=20 / \mathrm{mCherry}, 8 / \mathrm{hM} 3 \mathrm{Dq}$ ) Error bars are smaller than height of each data point. 

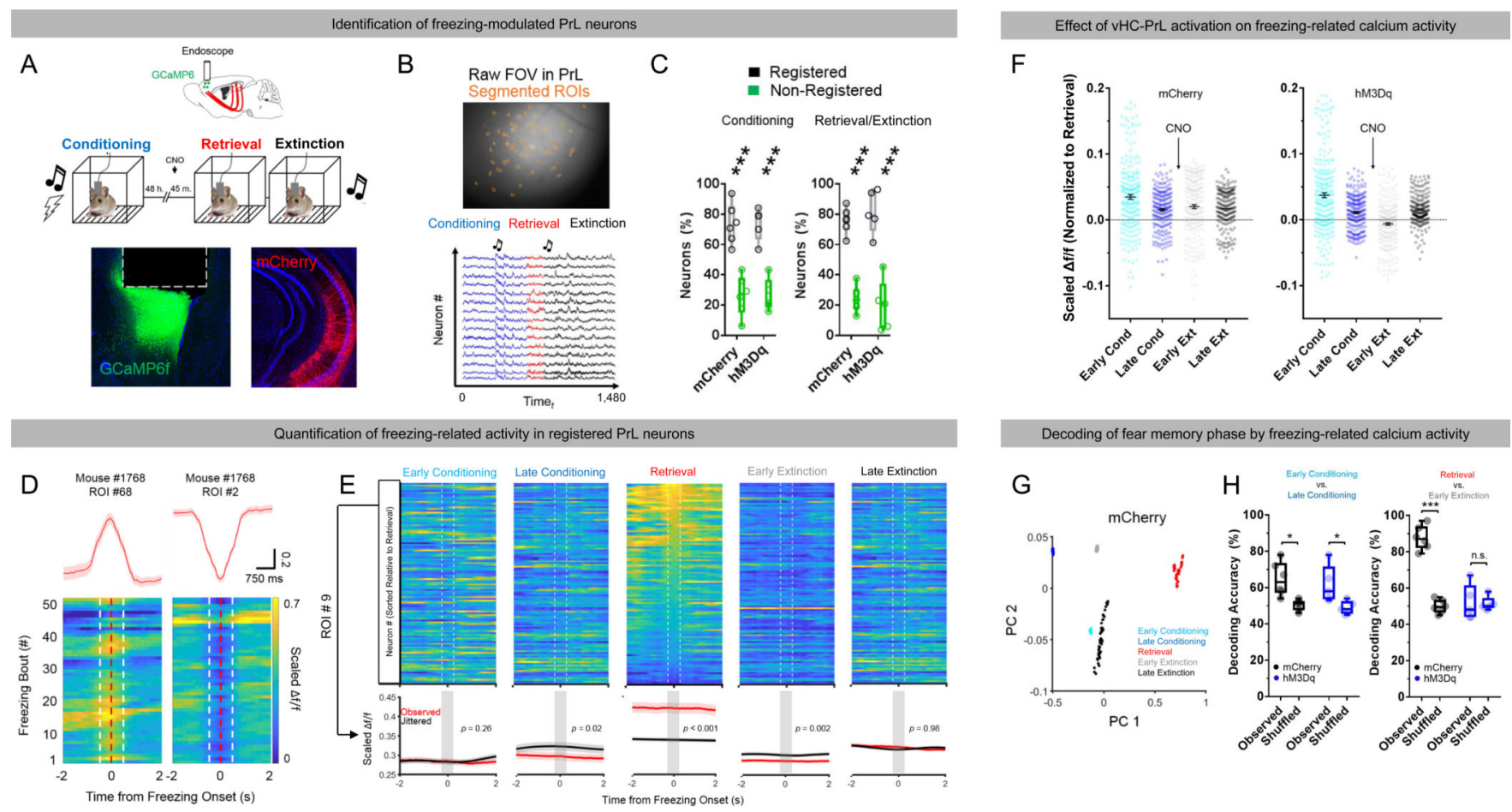

Figure 2.

vHC-PrL projectors alter population dynamics in the PrL during context retrieval and extinction training. (A) Schematic of experimental design. (Top) The excitatory DREADD receptor hM3Dq was expressed in vHC-PrL projectors, and GCaMP6f was expressed in the PrL for simultaneous imaging of PrL neurons during fear conditioning, context retrieval, and extinction training with a miniature one-photon microscope. (Bottom) Example confocal zprojection from the PrL showing GCaMP6f expression (green) and an endoscopic lens track (dashed line), and the vHC showing mCherry expression in projectors. (B) (Top) Example field of view from the PrL showing raw GCaMP6f fluorescence, with individual ROIs (single neurons) superimposed in orange. (Bottom) Example traces extracted from the PrL of one mouse registered across conditioning, retrieval, and extinction training. $(C) \mathrm{A}$ significant proportion of PrL neurons were able to be registered across fear phases in both mCherry and hM3Dq mice during both conditioning $((F(1,18)=82.64, p<0.0001$, main effect of registration category) and retrieval/extinction training $(\mathrm{F}(1,18)=120.4, p<0.0001$, main effect of registration category for 2 (condition) x 2 (registration category) ANOVA) (D) Example of a PrL neuron that shows increased activity ("freezing-increased"; left panel), and a PrL neuron that shows decreased activity ("freezing-decreased; right panel) during freezing bouts. (E) (Top row) Averaged activity from 86 simultaneously imaged PrL neurons from one mouse across early conditioning (pre-shock), late conditioning (post-shock), context retrieval, early extinction, and late extinction during freezing bouts. Neurons are sorted relative to their $\Delta \mathrm{f} / \mathrm{f}$ value during context retrieval freezing onset (dashed white lines). (Bottom row) Observed and jittered (peri-freezing) activity traces from one neuron in the population. This neuron varies its freezing- related activity as a function of fear epoch, reflecting the tendency of the population to shift its freezing-related activity patterns across fear learning, retrieval, and extinction. (F) On average, calcium activity during freezing 
bouts is higher during early conditioning, late conditioning, early extinction, and late extinction compared to retrieval (black dashed line at 0 on y-axis) in mCherry control mice (left panel). In hM3Dq mice, CNO injections caused freezing-related activity in PrL neurons to be more similar between early extinction and retrieval (closer to black dashed line), an effect that was not observed during other fear memory phases (right panel; $F(4)=15.69, p=$ 1.2ê-12, significant group $x$ fear memory phase interaction for linear mixed effects model). Calcium activity during fear phases was normalized to mean calcium activity during retrieval to make differences between retrieval and other fear phases more obvious. $(G)$ Principal components analysis (PCA) on population calcium activity during freezing reveals robust separation of clusters along the first two principal component axes as a function of fear memory phase in mCherry mice (each dot on graph represents calcium activity of all registered neurons in one time bin during freezing bouts). (H) Decoding accuracy of a linear classifier using freezing-related calcium activity to predict fear memory phase (observed) is significantly above chance (shuffled data) when distinguishing between early and late conditioning for both groups (left panel; mCherry $p=0.027$, hM3Dq $p=0.049$ ), but falls to chance levels when distinguishing between context retrieval and early extinction in the hM3Dq group (right panel; mCherry $p<0.0001$, hM3Dq $p=0.995$, Sidak's multiple comparison tests). 


\section{RNA-seq in MPFC after vHC-PrL projector stimulation}

A

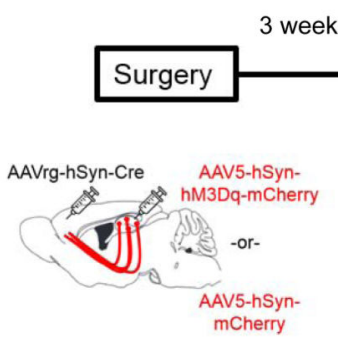

$\mathrm{CNO}+$ mCherry $\mathrm{CNO}+\mathrm{hM} 3 \mathrm{Dq}$ Saline + hM3Dq mCherry

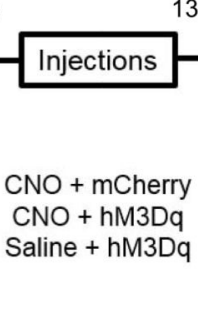

B

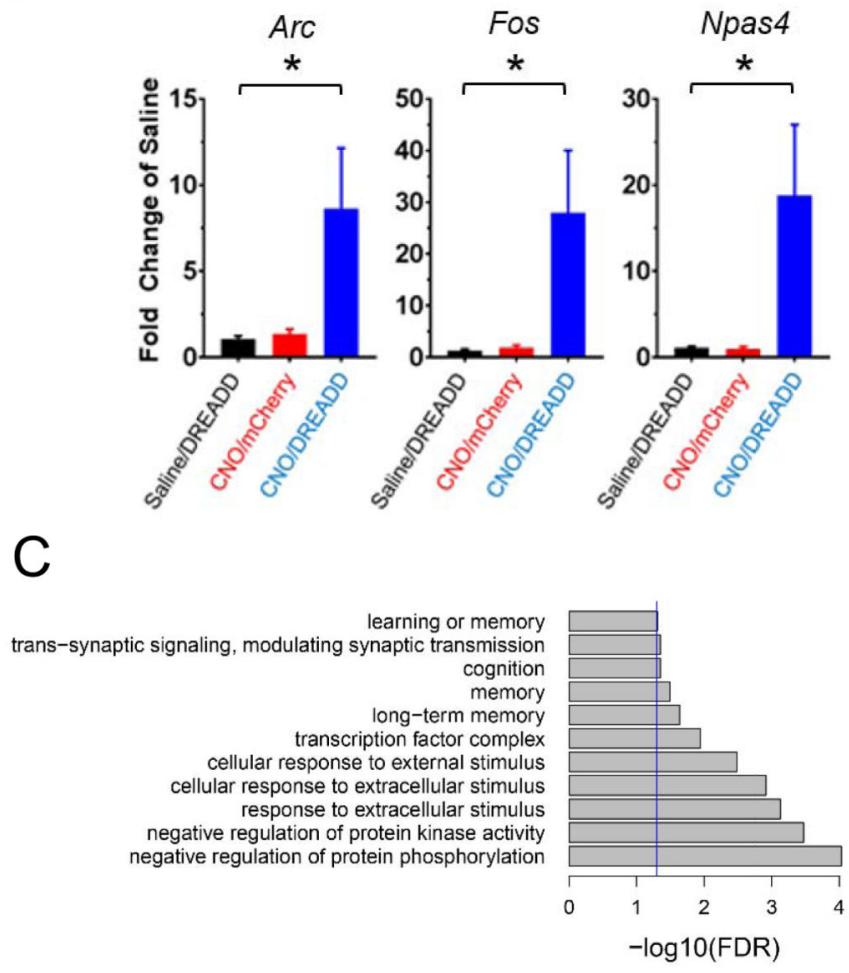

RNA Isolation qPCR Validation RNA Sequencing

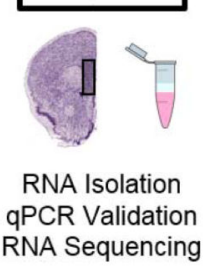

D

Depleted After Projector Stimulation Projector Stimulation

Figure 3.

Stimulation of vHC-PrL projectors alters activity-dependent gene expression in the PrL. $(A)$ Schematic of timeline and experimental design. (B) qPCR validates that immediate early genes (IEGs) are up- regulated in the PrL following stimulation of projectors, but not after CNO injections alone $(F(2,11)=3.991, p=0.0497$ for $\operatorname{Arc}, F(2,11)=4.213, p=0.0438$ for Fos, $F(2,11)=4.112, p=0.0464$ for $N p a s 4$, one-way ANOVAs). (C) GO enrichment analysis reveals significant up-regulation of genes that code for proteins necessary for learning, memory, cognition, long-term memory, and synaptic modulation following activation of projectors. (D) Volcano plot showing significantly differentially-expressed down-regulated (left of 0 on $\mathrm{x}$-axis) and up-regulated (right of 0 on $\mathrm{x}$-axis) genes (red dots; FDR $<0.1)$ in the PrL following projector activation. Genes related to BDNF-TrkB signaling are labeled with blue letters. $N$ trk $2 \log 2 \mathrm{FC}=0.4$, adjusted $p=0.044, B d n f \log 2 \mathrm{FC}$ 
$=0.84$, adjusted $p=0.014, A r c \log 2 \mathrm{FC}=0.67$, adjusted $p=7.21 \hat{\mathrm{e}}-5, N p t x 2 \log 2 \mathrm{FC}=0.85$, adjusted $p=0.0068$, Klf10 $\log 2 \mathrm{FC}=0.31$, adjusted $p=0.0007$, Adcyap $1 \log 2 \mathrm{FC}=1.65$, adjusted $p=1.08 \hat{\mathrm{e}}-9$. (E) Scatter plot showing directional overlap between differentiallyexpressed genes in our data set and differentially-expressed genes in a published data set from cultured neurons following BDNF application ( $p<0.0001 ; X^{2}$ test). 

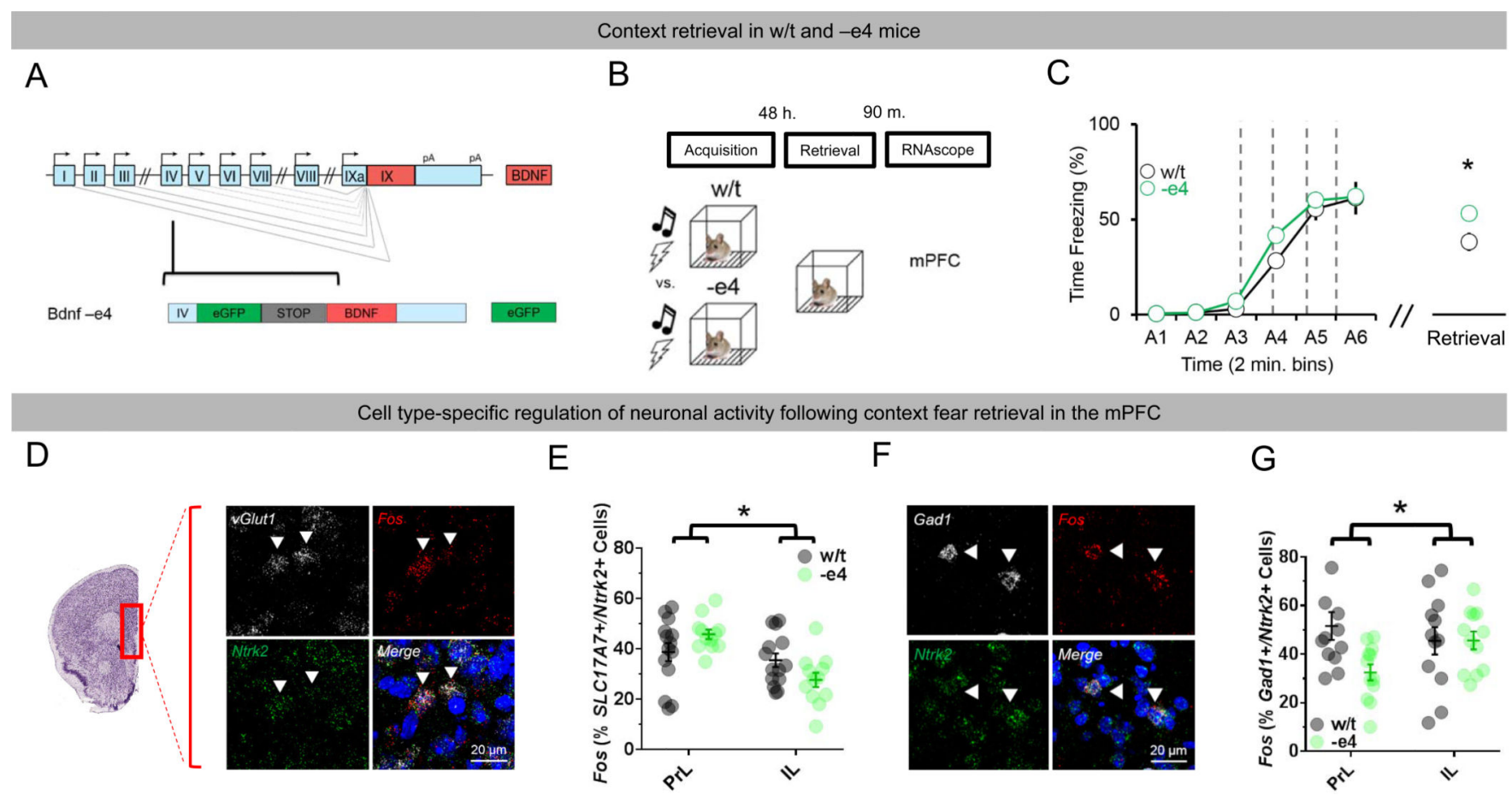

Figure 4.

Altered context fear retrieval and cell type-specific gene expression in the mPFC of mice with decreased activity-dependent BDNF production. (A) Schematic illustrating insertion of eGFP-STOP cassette downstream of promoter IV of the $B d n f$ gene in mice. This mutation leads to mice with decreased production of BDNF from promoter IV-derived transcripts (-e4 mice). (B) Schematic of experimental design and timeline for analysis of gene expression in the $\mathrm{mPFC}$ of $\mathrm{w} / \mathrm{t}$ and $-\mathrm{e} 4$ mice following context fear retrieval. $(C)$ Freezing during context retrieval, but not acquisition, is significantly higher in $-\mathrm{e} 4$ mice vs. w/t mice $(t(14)=2.855$, $p=0.0127$, unpaired t-test). (D) Example confocal z-projections from the mPFC showing co-expression of SIc17a7 (excitatory neurons), Fos (retrieval-activated neurons), and Ntrk2 (neurons modulated by BDNF signaling). (E) A significantly higher proportion of Ntrk2/ glutamatergic neurons in the PrL co-express retrieval-induced Fos in -e4 mice $(p=0.04$, Bonferroni-corrected t-test), while the opposite effect is observed in the IL ( $p=0.03$, Bonferroni-corrected t-test) of -e4 mice $(F(1,24)=6.099, p=0.021$, interaction for two-way ANOVA). (F) Example confocal z-projections from the mPFC showing co-expression of Gad1 (inhibitory neurons), Fos, and Ntrk2. (G) In contrast to Ntrk2/glutamatergic neurons, Ntrk2/GABAergic neurons in the PrL co-express Fos at lower levels in -e4 mice following context retrieval, while Ntrk2/GABAergic neurons in the IL co-express retrieval-induced Fos at similar levels in both genotypes $(F(1,22)=4.962, p=0.0365$, interaction for two-way ANOVA). 


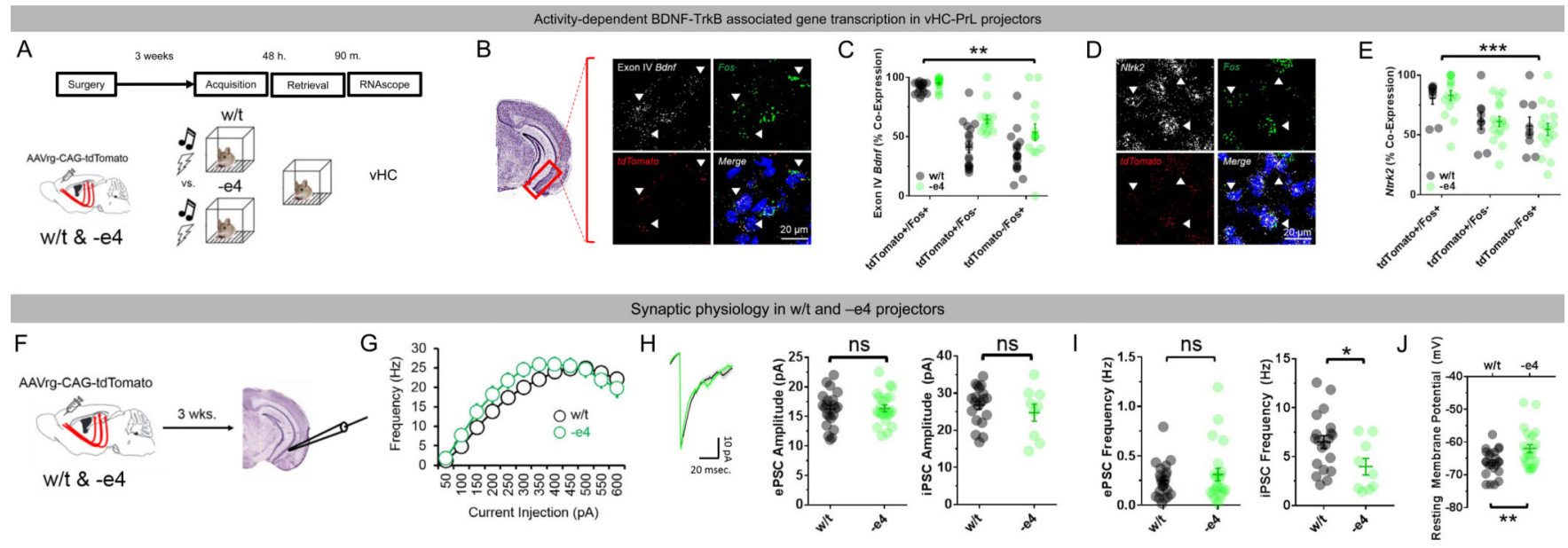

Figure 5.

BDNF-TrkB signaling in vHC-PrL projectors impacts their physiology and recruitment during context fear retrieval. (A) Schematic of experimental design and timeline for analysis of gene expression in the $\mathrm{vHC}$ of $\mathrm{w} / \mathrm{t}$ and $-\mathrm{e} 4$ mice following context fear retrieval. $(B)$ Example confocal z-projections from ventral CA1 showing co-expression of virally-induced tdTomato, context retrieval-induced Fos, and exon IV-containing Bdnf mRNA in individual neurons. $(C)$ Retrieval-activated projectors (tdTomato+/Fos+ neurons) are significantly enriched for exon IV-containing Bdnf, as compared to non-activated projectors (tdTomato+/ Fos-) and activated non- projectors (tdTomato-/Fos+) $(F(2,60)=147.6, p<0.0001$, main effect of cell type for 2 (genotype) x 3 (cell- type) mixed-factorial ANOVA). (D) Example confocal z-projections from ventral CA1 showing co-expression of tdTomato, Fos, and Ntrk2. (E) Retrieval-activated projectors are also significantly enriched for Ntrk 2 in both genotypes $(F(2,46)=32.57, p<0.0001$, main effect of cell type for 2 (genotype) $\mathrm{x} 3$ (celltype) mixed-factorial ANOVA). (F) Schematic of strategy for labeling and recording from vHC-PrL projectors ex vivo. $(G)$ Input- output curve showing no significant difference in firing rate as a function of current injection between wild/type (w/t) and -e4 mice. Error bars are smaller than height of each data point. $(H)$ Neither the amplitude of excitatory (left panel; $t(42)=0.17, p=0.87$ ) or inhibitory (right panel; $t(27)=0.92, p=0.37$, unpaired $\mathrm{t}$ tests) post-synaptic currents (ePSCs and iPSCs, respectively) onto projectors significantly differs between genotypes. (I) Frequency of iPSCs $(t(27)=2.224, p=0.0347)$, but not ePSCs $(t(42)=0.83, p=0.41$, unpaired t-tests), is significantly lower on -e4 projectors. $(J)$ Resting membrane potential is significantly more depolarized in -e4 projectors, as compared to $\mathrm{w} / \mathrm{t}$ projectors $(t(44)=2.917, p=0.0055$, unpaired t-test $)$. 

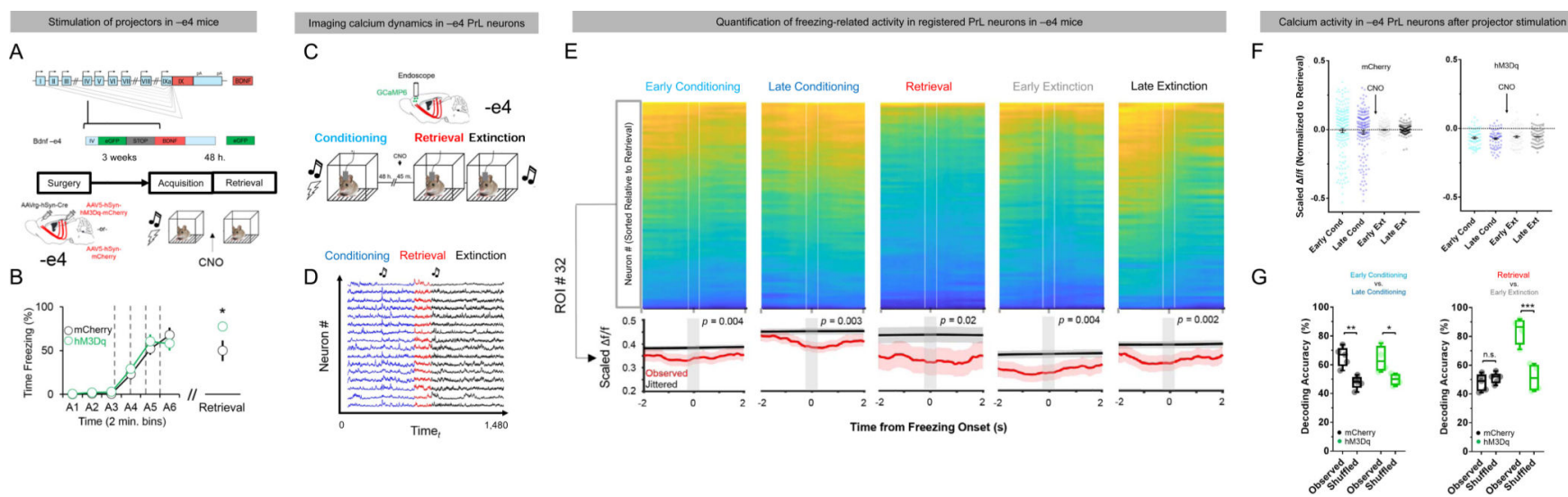

Figure 6.

Stimulation of vHC-PrL projectors induces instability in PrL populations during freezing and increases context fear memory expression in -e4 mice. (A) Schematic showing viral infection strategy for activation of vHC-PrL projectors prior to context retrieval in -e4 mice. (B) In contrast to $\mathrm{w} / \mathrm{t}$ mice, activation of projectors significantly increases freezing during context retrieval in $-\mathrm{e} 4$ mice $(t(15)=2.365, p=0.03$, unpaired t-test). (C) Cartoon of strategy for imaging calcium activity in PrL neurons following projector activation in -e4 mice. (D) Example calcium traces from 15 simultaneously imaged PrL neurons in an -e4 mouse registered across conditioning, context retrieval, and extinction training. (E) (Top row) Averaged activity from 66 simultaneously imaged PrL neurons from one -e4 mCherry mouse across early conditioning (pre-shock), late conditioning (post-shock), context retrieval, early extinction, and late extinction during freezing bouts. Neurons are sorted relative to their $\Delta \mathrm{f} / \mathrm{f}$ value during context retrieval freezing onset (dashed white lines). PrL population activity patterns during freezing remain similar between fear memory phases. (Bottom row) Observed and jittered (peri-freezing) activity traces from one neuron in the population. This neuron shows the same pattern of freezing-related activity during all fear memory phases. (F) On average, calcium activity during freezing bouts does not significantly differ between any fear memory phase and context retrieval (black dashed line at 0 on y-axis) in mCherry control -e4 mice (left panel). In hM3Dq mice, CNO injections caused calcium activity during context retrieval to become significantly higher relative to calcium activity during other fear memory phases (black dashed line, right panel; $F(4)=$ 4.52, $p=0.001$, significant group $\mathrm{x}$ fear memory phase interaction for linear mixed effects model). (G) Decoding accuracy of a linear classifier using freezing-related calcium activity to predict fear memory phase (observed) is significantly above chance (shuffled data) when distinguishing between early and late conditioning for both groups (left panel; mCherry $p=$ 0.002 , hM3Dq $p=0.012$ ), but falls to chance levels when distinguishing between context retrieval and early extinction in the mCherry group (right panel; mCherry $p=0.74, \mathrm{hM} 3 \mathrm{Dq}$ $p=0.0004$, Sidak's multiple comparison tests). 

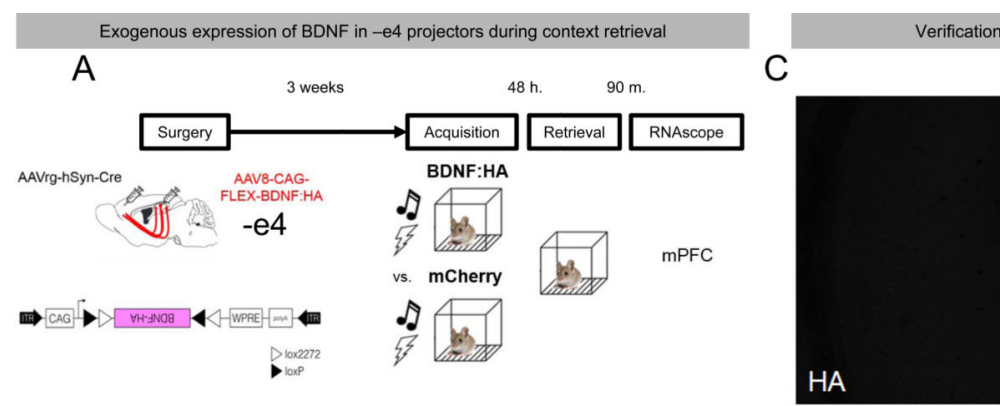

erification of BDNF:HA expression in ventral CA1

B
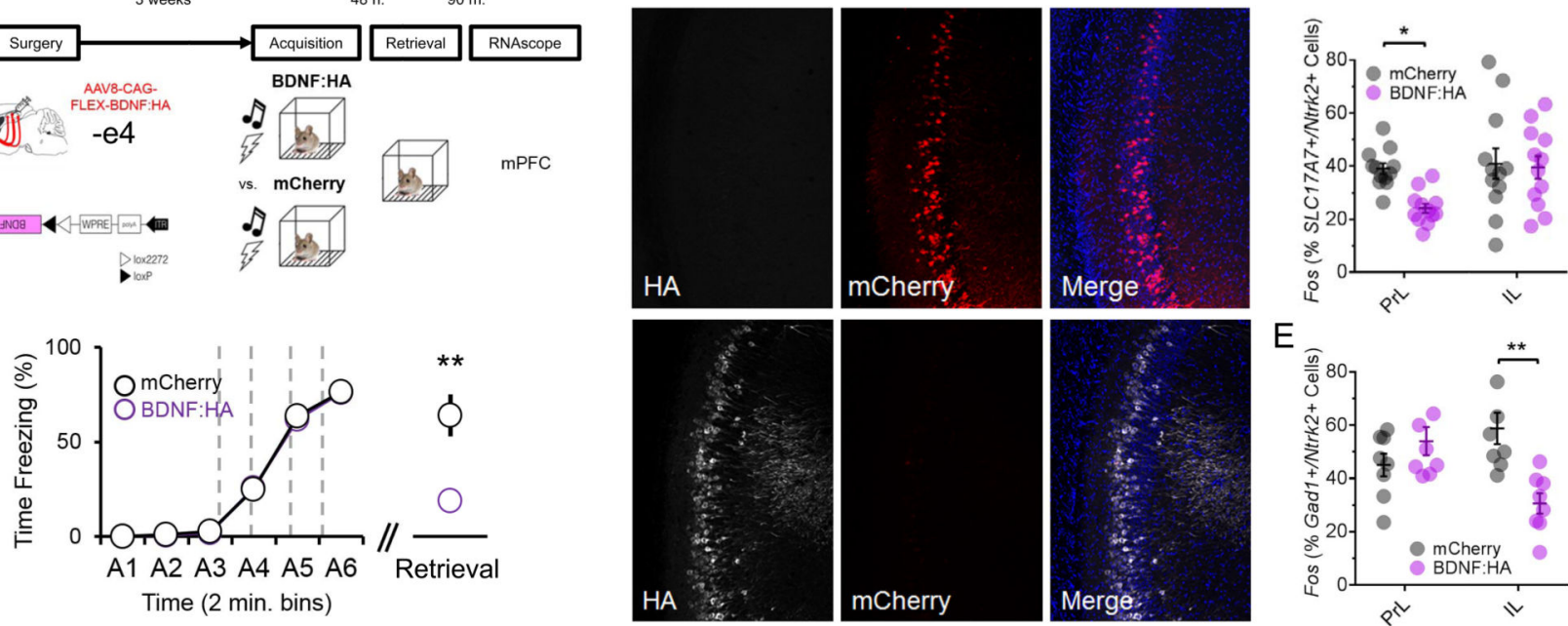

Figure 7.

Exogenous expression of BDNF protein in vHC-PrL projectors reverses fear-related behavioral and molecular phenotypes in -e4 mice. (A) Schematic of timeline and experimental design for viral over-expression of BDNF in -e4 projectors. Bottom panel shows sequence for AAV8-CAG-FLEX-BDNF:HA construct. (B) Over- expression of $\mathrm{BDNF}$ in projectors reduces freezing in -e4 mice during context retrieval, but not fear acquisition $(t(14)=3.753, p=0.0021$, unpaired t-test). Error bars are smaller than height of each data point. (C) Example confocal z-projections from ventral CA1 showing mCherry expression in control -e4 animals, and immunofluorescence for HA protein in BDNF:HA animals. (D) BDNF over-expression in -e4 projectors reduces the proportion of Ntrk2l glutamatergic neurons that co-express Fos in the $\operatorname{PrL}(F(1,22)=5.423, p=0.0295$, interaction for two-way ANOVA). ( $E$ ) BDNF over-expression in -e4 projectors reduces the proportion of $N$ trk2/GABAergic neurons that co-express Fos in the $\operatorname{IL}(F(1,14)=13.7, p=$ 0.0024 , interaction for two-way ANOVA). 